\title{
Neuropathology of the Guillain-Mollaret Triangle (Dentato-Rubro-Olivary Network) in Sudden Unexplained Perinatal Death and SIDS
}

\author{
Anna Maria Lavezzi*, Melissa Corna, Luigi Matturri and Franco Santoro
}

\author{
Department of Surgical, Reconstructive and Diagnostic Sciences - "Lino Rossi" Research Center for the study and \\ prevention of unexpected perinatal death and SIDS, University of Milan, Italy
}

\begin{abstract}
The present study was undertaken to evaluate the possible alterations of the triangle of Guillain and Mollaret (G-Mt), a neuronal brainstem/cerebellum network (from the dentate nucleus to red nucleus and inferior olivary nucleus) already known for its involvement in the pathogenesis of the palatal myoclonus, in sudden unexplained perinatal and infant death. In 44 cases of perinatal and infant death victims, aged from 26 gestational weeks to 10 months of life, we investigated, besides the histological morphology of the three nuclei, the c-fos and apoptotic expression, as well as the possible effects elicited by maternal cigarette smoking.

A significant increase of lesions (hypoplasia and/or increased c-fos and apoptotic neuronal immunopositivity) of the three nuclei was found in unexplained death victims, compared with age-matched controls. These alterations were related to maternal cigarette smoking habit.

We postulated that the G-Mt is an important network involved in the pathogenesis of a wide spectrum of pathological manifestations and that maternal smoking during pregnancy can exert an adverse influence on this complex, even leading to sudden death in vulnerable periods of perinatal or infant development.
\end{abstract}

Keywords: Dentate nucleus, Inferior olivary nucleus, Red nucleus, Palatal myoclonus, Maternal cigarette smoking, SIDS, Unexplained perinatal death.

\section{INTRODUCTION}

Early studies on palatal myoclonus ascribed the cause of this pathologic manifestation, characterised by rhythmic involuntary contractions of the palatal musculature, to neuronal lesions affecting the dentato-rubro-olivary pathway or Guillain-Mollaret triangle (G-Mt) [1-7]. This is a functional circuit connecting the dentate nucleus (DN) of the cerebellum of one side with the red nucleus (RN) and the inferior olivary nucleus (ION) on the other side, via the superior cerebellar peduncle, the central tegmental tract and the inferior cerebellar peduncle, respectively (Fig. 1).

We previously highlighted the vulnerability of the ION and of the DN to hypoxia, prevalently expressed by increased neuronal apoptosis and c-fos expression, in unexplained perinatal and infant death victims [8]. Since even the $\mathrm{RN}$ may play a part in the autonomic responses which occur in hypoxic conditions $[9,10]$, we aimed to evaluate in the present study the possible involvement of the G-Mt in sudden fetal and infant death, like the alterations in nuclei and/or structures of the brainstem and cerebellum checking vital functions, by us observed in these pathologies [11-16].

In particular, we investigated in a large group of perinatal and infant death victims, aged from 26 gestational weeks to 10 months of life, besides the morphological structure of the ION, RN and DN in histological sections of medulla oblon-

*Address correspondence to this author at the "Lino Rossi" Research Center, Department of Surgical, Reconstructive and Diagnostic Sciences, University of Milan, Via della Commenda, 19, 20122 Milan, Italy; Tel: +39-0250320821; Fax: +39-02-50320823; E-mail: anna.lavezzi@unimi.it gata, midbrain and cerebellum, respectively, the immunoexpression of c-fos proto-oncogene and apoptosis, biomarkers frequently involved in neuronal responses to hypoxic injuries [17-20].

In addition, the observation in our previous studies of a significantly increased incidence of structural and/or functional alterations of different brainstem nuclei in stillborns and sudden infant death syndrome (SIDS) victims with smoker mothers $[8,21,22]$, prompted us to verify whether prenatal absorption of nicotine could also be related to developmental abnormalities of the G-Mt.

\section{MATERIALS AND METHODS}

In total, 44 brains were collected from 22 perinatal victims (15 fetuses, aged 26-39 gestational weeks, 7 newborns who died within the first days of life) and 22 infants aged 110 months (mean age: 3 postnatal months). There were 26 males and 18 females. Pregnancy in all cases had run a normal course. None of the mothers had any significant pathology.

The victims were subjected to a complete autopsy, including examination of the placental disk, umbilical cord and membranes in fetal deaths. In all cases an in-depth histological examination of the autonomic nervous system was made, according to the protocol routinely followed by the "Lino Rossi" Research Center of the Milan University $[23,24]$.

From the brainstem and cerebellum, transverse serial sections were made at intervals of $60 \mu \mathrm{m}$.

For each level ten $5 \mu \mathrm{m}$ sections were obtained, two of which were stained for histological examination using hema- 


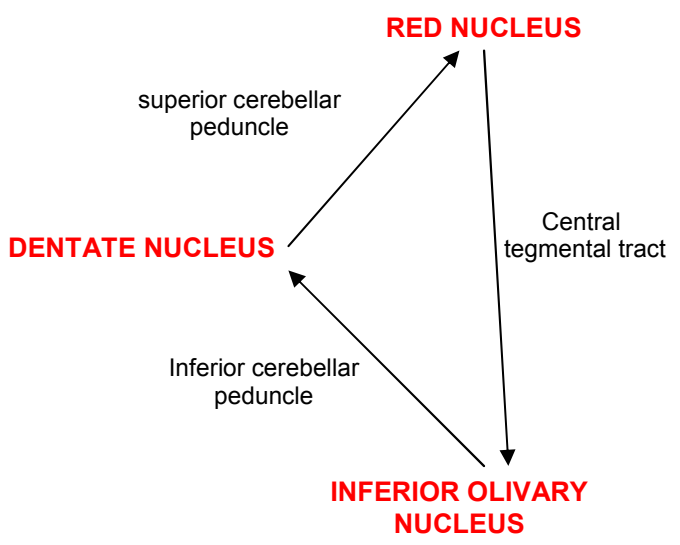

Fig. (1). Drawing showing the triangle of Guillain and Mollaret.

toxylin-eosin and Klüver-Barrera stains, and two additional sections were subjected to immunohistochemistry of the apoptosis and c-fos expression. The remaining sections were saved for further investigations.

The examination of the inferior olivary nucleus, the red nucleus and the dentate nucleus, the target of this study, was performed in all the cases at the same levels of the medulla oblongata (obex), mesencephalon (superior colliculus) and at the midline of either cerebellar hemispheres. These histological sections also allowed to easily analyze further important structures (dorsal motor vagus, tractus solitarius, hypoglossus, ambiguous, pre-Bötzinger and arcuate nuclei in the medulla oblongata; Kölliker-Fuse nucleus and substantia nigra in the mesencephalon; fastigial, globose, emboliform nuclei and cortex layers in the cerebellum).

In 28 cases, 10 fetuses, 4 newborns and 14 infants, after the in-depth examination, no cause of death was found. These cases were defined Sudden Intrauterine Unexplained Death (SIUD), Sudden Neonatal Unexplained Death (SNUD), and Sudden Infant Death Syndrome (SIDS), respectively.

In the remaining 16 cases, 5 fetuses, 3 newborns and 8 infants, a precise cause of death was formulated at autopsy, with no evidence of neuropathological lesions; these constituted the control cases. Specific diagnoses among the fetal and neonatal deaths included: chorioamnionitis $(n=2)$ and congenital heart disease $(n=6)$. Specific diagnoses among the infant deaths were: congenital heart disease $(n=4)$, severe bronchopneumonia $(n=3)$ and pericarditis $(n=1)$.

While taking the medical history, the mother was asked for information about any smoking habit before, during and after pregnancy. Smoking habit was assigned to two categories (smokers vs. nonsmokers). Overall, 22 of the 44 mothers (50\%) were smokers of more than 3 cigarettes a day already before the onset of the pregnancy, and 22 were non smokers. Eighteen women in the smoker group were mothers of a victim of sudden unexplained death.

\section{Immunohistochemical Methods}

In order to analyze the immuno-expression of c-fos, we used specific primary antibody after applying the avidinbiotin-peroxidase technique, in conformity with conventional immunohistochemical procedures. Immunohistochemical visualization of apoptotic cells was obtained by the TUNEL method (TdT-mediated dUTP-biotin nick end labeling). A detailed description of the immunohistochemical procedures that we applied, including the immunopositivity evaluation, is available in our previous works $[8,12,14]$.

Both morphological and immunohistochemical analyses were performed by at least two independent and blinded observers. All the results were compared to evaluate the interobserver reproducibility. In cases of discordance among the investigators, the case was reviewed and discussed until a unanimous result was obtained.

\section{Statistical Analysis}

Data were analyzed using the SPSS statistical software package (version 11.0; SPSS Inc., Chicago, IL, USA). Fisher's exact test was applied for comparisons. P values $<0.05$ were considered statistically significant.

\section{RESULTS}

\section{Morphological Examination of the G-Mt}

Histological examination performed on brainstems and cerebella of the 44 victims demonstrated, in most of the cases, particularly in the subjects who died of known cause, a normal morphology, consistently with the gestational age in fetal deaths, of the inferior olivary, dentate and red nuclei.

Nevertheless, developmental cytoarchitectural abnormalities, represented by hypoplasia of the three nuclei (ION, DN and RN), were found in 7 of the 14 SIDS cases (50\%), in 2 of the SNUD and in 2 of the 10 SIUD victims (22\%).

The diagnosis of DN hypoplasia was expressed when this nucleus showed small-size, simplified structure, with poor convolutions, and decreased neuronal density compared with the DN morphology of age- matched controls.

The hypoplasia of the ION was defined by lack of both medial and dorsal olivary accessory nuclei and reduced undulation of the principal olivary nucleus (Fig. 2).

The hypoplasia of the RN was diagnosed in absence of the large neurons of the caudal magnocellular portion (Fig. 3). 
A

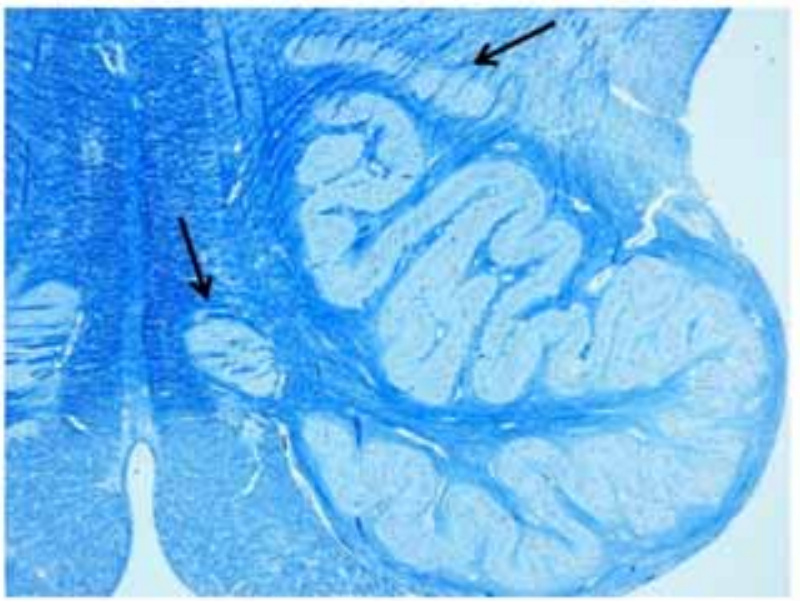

B

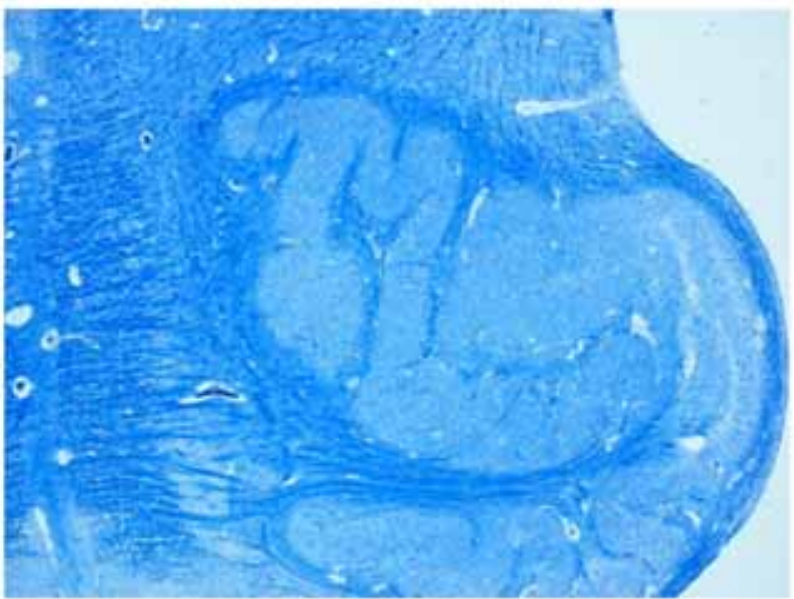

Fig. (2). Inferior olivary nucleus.

A) Normal structure in a control case (female, aged 2 months). Arrows point to accessory subnuclei (dorsal and medial, respectively).

B) Hypoplasia in a case of SIDS (male, aged 4 months) with lack of both the medial and dorsal accessory nuclei.

Klüver-Barrera stain. Magnification: 4x.

A
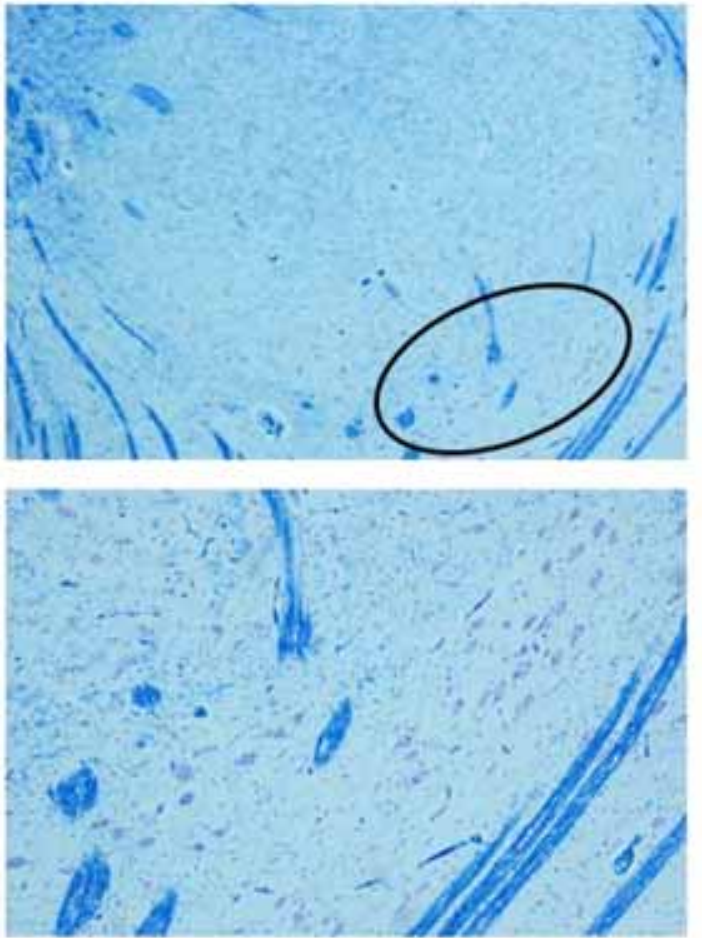

B
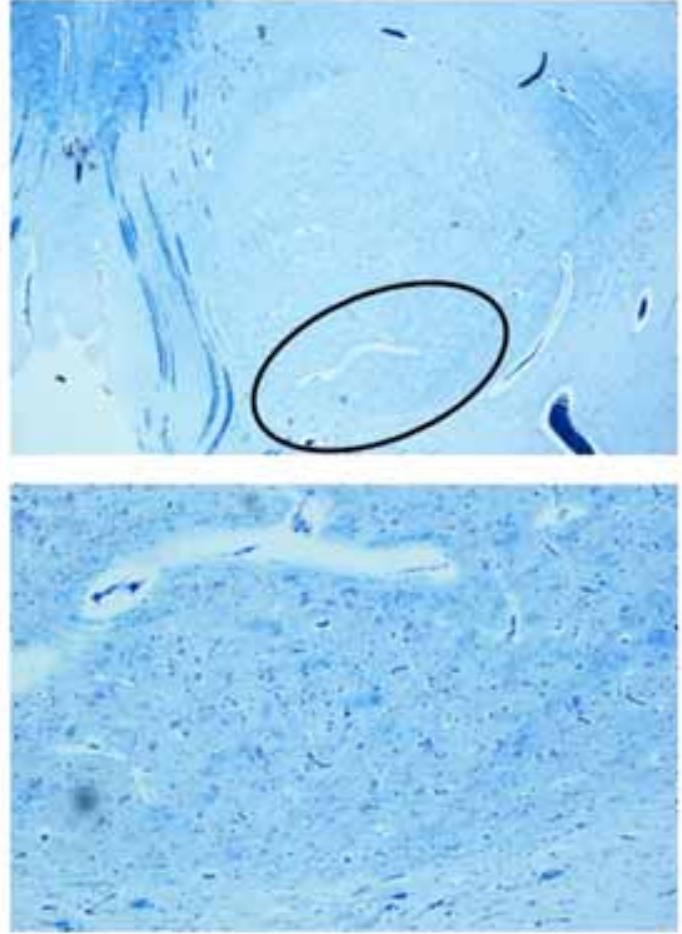

Fig. (3). Red nucleus.

A) Normal structure in a control case (male, aged 4 months). Below, higher magnification of the encircled area in the upper image with large neurons of the caudal magnocellular portion.

B) Hypoplasia in a case of SIDS (male, aged 3 months). Below, higher magnification of the encircled area in the upper image with lack of large neurons that are typical of the caudal magnocellular portion.

Klüver-Barrera stain. Magnification: in the upper images 4x; below 10x.

These morphological alterations were not seen in victims of the control group (statistical significance of sudden death group vs. control group: $\mathrm{p}<0.01)$.

\section{Immunohistochemical Examination of the G-Mt}

c-fos immunohistochemistry - In 10 sudden death victims ( 5 fetuses, 1 newborn and 4 infants), more than $80 \%$ of the 
A

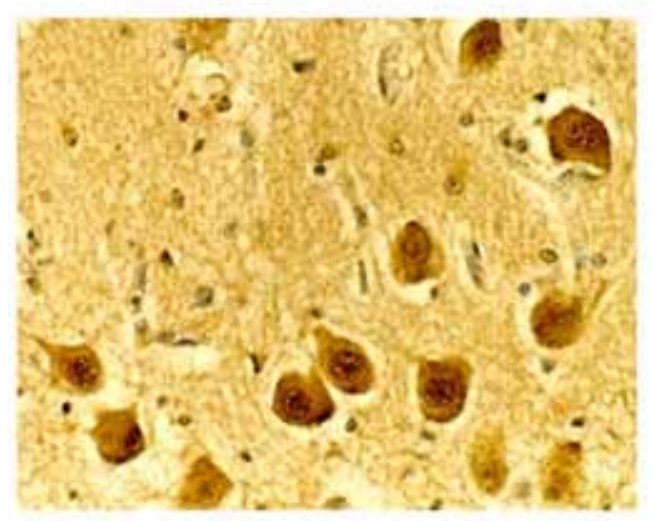

B

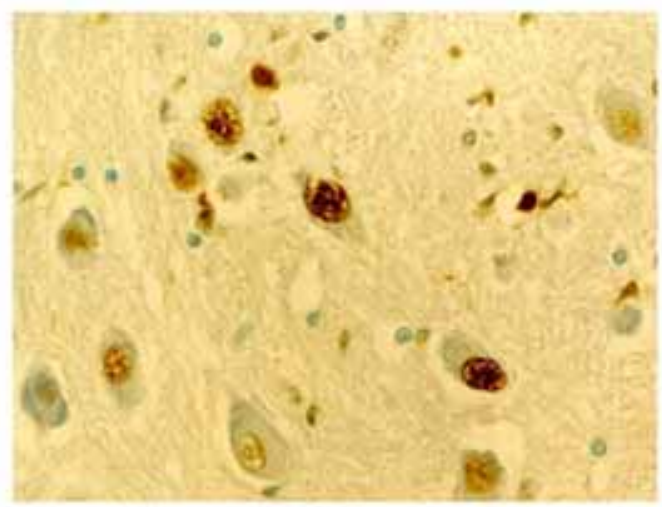

Fig. (4). Immunohistochemistry.

A) c-fos immunopositivity of neurons in the cerebellar dentate nucleus of a sudden infant death syndrome victim, 3 months-old.

B) Apoptotic neurons in the inferior olivary nucleus in a case of sudden intrauterine unexplained fetal death (aged 38 gestational weeks). Magnification: 40x.

neurons of the three nuclei were intensely c-fos labeled. In the same nuclei of the other cases c-fos expression was negative or scarcely positive ( $<25 \%$ of positive cells) (Fig. 4A).

\section{Apoptosis Immunohistochemistry}

We observed high percentages (ranging from $70 \%$ to 90\%) of apoptotic neurons (TUNEL-positive cells with highly condensed chromatin, various nuclear fragmentation stages and apoptotic bodies) in the three analyzed nuclei of 5 sudden perinatal deaths, 7 SIDS victims and two cases in the infant control group, both of whom died of pneumonia (Fig. 4B). Neuronal apoptotic values, ranging from 0 to $20 \%$, were found among the other brainstem and cerebellum nuclei.

High c-fos and apoptotic expressions were present even in hypoplastic DN, RN and ION observed in 8 of the 11 unexplained deaths.

Table 1 summarizes the G-Mt alterations observed in perinatal and infant death victims of the study. Statistical evaluation suggested that sudden unexplained death was significantly associated with G-Mt neuropathological alterations.

In the great part of SIUD, SNUD and SIDS victims with developmental abnormalities of the G-Mt, we also observed morphological alterations of different brainstem and cerebellum structures (precisely, hypoplasia/agenesis of the arcuate nucleus, pre-Bötzinger nucleus, raphé obscurus nucleus, parafacial nucleus and cerebellar cortex delayed maturation). The most frequent association was between G-Mt alterations and hypodevelopment of the medullary arcuate nucleus in SIDS victims, hypoplasia of the pontine parafacial nucleus in sudden fetal deaths.

Finally, we correlated all the findings with the mother's smoking habit. A significant relation was evident between maternal smoking, sudden unexplained deaths and alterations of the G-Mt. In fact, 12 of the 18 unexplained death victims with smoking mother showed neuropathological findings (hypoplasia and/or increased c-fos and apoptotic expression) of the G-Mt ( $\mathrm{p}<0.01)$.

\section{DISCUSSION}

The important role of the G-Mt alterations in the pathogenesis of the palatal myoclonus, a rare movement disorder consisting of rhythmic involuntary jerky movements of the soft palatal musculature, has been outlined by several authors, even if the pathomechanism generating these rhythmic contractions remained unexplained [1-7].

In this study we demonstrated that lesions that disrupt this patway are not exclusive of the palatal tremor. The results documented in fact significant increase of biological and cytoarchitectural alterations of all the three nuclei of the G-Mt in sudden unexplained fetal and infant death victims, compared with age-matched controls.

In particular, we observed major defects represented by hypoplasia of the DN, ION and RN, above all in SIDS victims, and subtle alterations represented by high neuronal c-fos and apoptotic expression.

Table 1. Alterations of the G-Mt in Perinatal and Infant Deaths

\begin{tabular}{|c|c|c|c|}
\hline $\begin{array}{c}\text { DN, ION, RN } \\
\text { Neuropathological Alterations }\end{array}$ & $\begin{array}{c}\text { Sudden Unexplained Death (SIUD + SNUD + SIDS) } \\
\mathbf{n = 2 8}\end{array}$ & $\begin{array}{c}\text { Control Perinatal and Infant Death } \\
\mathbf{n}=\mathbf{1 6}\end{array}$ & \begin{tabular}{c} 
p Value \\
\hline \hline hypoplasia
\end{tabular} \\
\hline \hline c-fos positivity* & 11 & - & 0.035 \\
\hline Apoptosis positivity* & 10 & - & 0.048 \\
\hline
\end{tabular}

SIUD = sudden intrauterine unexplained death; SNUD = sudden neonatal unexplained death; SIDS = sudden infant death syndrome.

$*=>25 \%$ of immunopositive neurons. 
These developmental lesions can affect the reactiveness and the autonomic compensatory responses of the G-M nuclei to low oxygen conditions. Besides, Tai and Truong [25] demonstrated that cerebral hypoxic insults can also be one of the main causes of myoclonus in animal models, by using Fos protein as a marker of neuronal activity.

The proto-oncogene c-fos in fact is the first gene activated by noxious signals, particularly in presence of hypoxia $[17,19]$. Therefore, immunohistochemical labeling of the fos-corresponding protein in activated neurons has been recognized as an important component of the neuronal response to brain injuries. Experimental works have demonstrated in particular that exposure to hypoxia markedly increases fosimmunoreactivity within the brainstem and cerebellar deep nuclei [26-28]. Likewise, several Authors have provided evidence, on the basis of TUNEL-staining studies in rodents, that serious DNA fragmentation associated with apoptosis is observable in response to hypoxic injury in the central nervous system neurons, particularly in the brainstem and cerebellum $[29,30]$.

Thus we detain that lesions in the G-Mt may determine a variety of pathological manifestations which, even if quite different, recognize as common etiology the hypoxia. All these pathologies can be related to a defective muscular contraction. Soft palate, and sometimes eye, face and throat muscles are involved in palatal myoclonus [2,4,31]; the upper airway muscles in sudden unexplained death.

The nuclei of the G-Mt, above all the DN, are in fact critical structures for the control of the somatic muscle contraction, including the respiratory muscles [32,33]. The brainstem and cerebellar nuclei damage following low oxygen delivery may contribute to loss of coordination of upper airway muscle activity, thus preventing eupneic breathing and leading to fatal outcome.

It can be reasonably understandable that alterations of the G-M nuclei may give rise to respiratory inhibition after birth, and consequently to sudden neonatal and infant death. Nevertheless it is difficult to explain how breathing alterations can lead to death during intrauterine life.

We know that periodic fetal breathing regulates the release of tracheal fluid in the lung, and consequently alveolar expansion, to favor lung development [34,35]. Nevertheless, defective intrauterine respiratory movements would not be sufficient to justify fetal loss.

One possibility that we strongly support, is that the G-Mt in fetal life, likewise to other brainstem and cerebellum structures [11-16], participates in the modulation of all the vital functions. Consequently, disruption of the ascending and descending patways between mesencephalon/ cerebellum/medulla can produce severe dysregulation of the autonomic nervous system, triggering the death mechanism before the birth.

Inhalation of smoke is the possible risk factor for hypoxic events that may contribute to G-Mt alterations in perinatal period. In cases of maternal smoking during pregnancy, carbon monoxide, a gaseous combustion product of nicotine, may readily cross the placenta, where it binds to hemoglobin, by passive diffusion. Consequently, carboxyhemoglobin inhibits oxygen release into the fetal tissues causing hypoxia, especially in the most susceptible organs, including the brain. Nicotine can thus give rise to damage in neuronal networks regarded as selectively vulnerable [36-38].

In agreement with these considerations is the observation in this study of a significant relation between maternal smoking and biomorphological alterations of the G-Mt in sudden unexplained perinatal and infant death.

We conclude that the G-Mt constitutes an important network involved in the pathogenesis of a wide spectrum of pathological manifestations. The consequences of developmental alterations of the G-Mt can be minor such as in palatal tremor, less soft in cerebellar atrophy, resulting from olivo-dentate fiber disruption [39], or very serious leading to unexpected death in vulnerable periods of the perinatal life.

\section{REFERENCES}

[1] Deuschl G, Mischke G, Schenck E, Schulte-Mönting J, Lücking $\mathrm{CH}$. Symptomatic and essential rhythmic palatal myoclonus. Brain 1990; 113: 1645-72.

[2] Dubinsky RM, Hallett M. Palatal myoclonus and facial involvement in other types of myoclonus. Adv Neurol 1988; 49: 263-78.

[3] Goyal M, Versnick E, Tuit P, et al. Hypertrophic olivary degeneration: metaanalysis of the temporal evolution of MR findings. AJNR Am J Neuroradiol 2000; 21: 1073-7.

[4] Guillain G, Mollaret P. Deux cas de myoclonies synchrones et rythmees velo-pharygolaryngo-oculo-diaphragmatiques: le probleme anatomique et physio-pathologique de ce syndrome. Rev Neurol 1931; 2: 545-66.

[5] Hong S, Leigh RJ, Zee DS, Optical LM. Inferior olive hypertrophy and cerebellar learning are both needed to explain ocular oscillations in oculopalatal tremor. Prog Brain Res 2008; 171: 219-26.

[6] Katsuse O, Dickson DW. Inferior olivary hypertrophy is uncommon in progressive supranuclear palsy. Acta Neuropathol 2004; 108: 143-6.

[7] Lapresle J. Rhythmic palatal myoclonus and the dentato-olivary pathway. J Neurol 1979; 220: 223-30.

[8] Lavezzi AM, Ottaviani G, Mauri M, Matturri L. Biopathology of the olivocerebellar network in sudden unexplained perinatal and sudden infant death syndrome related to maternal cigarette smoking. Neurol Res 2007; 29: 525-32.

[9] Ackland GL, Noble R, Hanson MA. Red nucleus inhibits breathing during hypoxia in neonates.Respir Physiol 1997; 110: 251-60.

[10] Waites BA, Ackland GL, Noble R, Hanson MA. Red nucleus abolish the biphasic respiratory response to isocapnic hypoxia in decerebrate rabbits. J Physiol 1996; 495: 217-25.

[11] Lavezzi AM, Ottaviani G, Ballabio G, Rossi L, Matturri L. Preliminary study on the cytoarchitecture of the human parabrachial/Kölliker-Fuse complex with reference to sudden infant death syndrome and sudden intrauterine unexplained death. Pediatr Dev Pathol 2004; 7: 171-9.

[12] Lavezzi AM, Ottaviani G, Matturri L. Role of somatostatin and apoptosis in breathing control in sudden perinatal and infant unexplained death. Clin Neuropathol 2004; 23: 304-10.

[13] Lavezzi AM, Ottaviani G, Mingrone R, Rossi L, Matturri L. Analysis of the human locus coeruleus in perinatal and infant sudden unexplained death: possible role of the cigarette smoking in the development of this nucleus. Brain Res Dev Brain Res 2005; 154: 71-80.

[14] Lavezzi AM, Ottaviani G, Mauri M, Matturri L. Alterations of biological features of the cerebellum in sudden perinatal and infant death. Curr Mol Med 2006; 6: 429-35.

[15] Lavezzi AM, Matturri L. Functional neuroanatomy of the human pre-Bötzinger complex with particular reference to sudden unexplained perinatal and infant death. Neuropathology 2008; 28: 10-6.

[16] Lavezzi AM, Matturri L. Hypoplasia of the parafacial/facial complex: a very frequenty finding in sudden unexplained fetal death. Open Neurosci J 2008; 2: 1-5.

[17] Herrera DG, Robertson HA. Activation of c-fos in the brain. Prog Neurobiol 1996; 50: 83-107.

[18] Machaalani R, Waters KA. Increased neuronal cell death after intermittent hypercapnic hypoxia in the developing piglet brainstem. Brain Res 2003; 985: 127-34. 
[19] Sica AL, Greenberg HE, Scharf SM, Ruggiero DA. Immediateearly gene expression in cerebral cortex following exposure to chronic-intermittent hypoxia. Brain Res 2000; 870: 204-10.

[20] Yue X, Mehmet H, Penrice J, et al. Apoptosis and necrosis in the newborn piglet brain following transient cerebral hypoxiaischaemia. Neuropathol Appl Neurobiol 1997; 23: 16-25.

[21] Lavezzi AM, Ottaviani G, Mauri M, Matturri L. Hypoplasia of the arcuate nucleus and maternal smoking during pregnancy, in perinatal and infant sudden unexpected death. Neuropathology 2003; 24: 284-9.

[22] Lavezzi AM, Ottaviani G, Matturri L. Adverse effects of prenatal tabacco smoke exposure on biological parameters of the developing brainstem. Neurobiol Dis 2005; 20: 601-7.

[23] Matturri L, Ottaviani G, Lavezzi AM. Techniques and criteria in pathologic and forensic-medical diagnostics of sudden unexpected infant and perinatal death. Am J Clin Pathol 2005; 124: 259-68.

[24] Matturri L, Ottaviani G, Lavezzi AM. Guidelines for neuropathologic diagnostics of perinatal unexpected loss and sudden infant death sindrome (SIDS): a technical protocol. Virchows Arch 2008; 452: 19-25.

[25] Tai KK, Truong DD. Post-hypoxic myoclonus induces Fos expression in the reticular thalamic nucleus and neurons in the brainstem. Brain Res 2005; 1059: 122-8.

[26] Hirooka Y, Polson JW, Potts PD, Dampney RA. Hypoxia-induced Fos expression in neurons projecting to the pressor region in the rostral ventrolateral medulla. Neuroscience 1997; 80: 1209-24.

[27] Nogami M, Takatsu A, Endo N, Ishiyama I. Immunohistochemical localization of c-fos in the nuclei of the medulla oblongata in relation to asphyxia. Int J Legal Med 1993; 112: 351-4.

[28] Xu F, Gibson T, Zhou T, Zhou D, Frazier DT. Hypoxia activates neurons in the fastigial nucleus of the rat cerebellum (Abstract). FASEB J 2000; J14: A494.
[29] David JC, Grongnet JF. Effect of hypoxia on DNA fragmentation in different brain regions of the newborn piglet. Mol Reprod Dev 2000; 57: 153-8.

[30] Peng JHF, Feng Y, LeBlanc MH, Rhodes PG, Parker JC. Apoptosis and necrosis in developing cerebellum and brainstem induced after focal cerebral hypoxic-ischemic injury. Dev Brain Res 2005; 156: 87-92.

[31] Bender MB, Nathanson M, Gordon GG. Myoclonus of the muscles of the eye, face and throat. Arch Neurol Psychiatry 1952; 67: 4458 .

[32] Gruart A, Maria J. Respiration-related neurons recorded in the deep cerebellar nuclei of the alert cat. Neuroreport 1993; 3: 365-8.

[33] Xu F, Frazier DT. Role of the cerebellar deep nuclei in respiratory modulation. Cerebellum 2000; 1: 35-40.

[34] Boddy K, Dawes GS. Fetal breathing. Br Med Bull 1975; 31: 3-7.

[35] Harding R, Bocking AD, Sigger JN. Influence of upper respiratory tract on liquid flow to and from fetal lungs. J Physiol 1986; 61: 6871.

[36] Cole PV, Hawkins LH, Roberts D. Smoking during pregnancy and its effect on the fetus. J Obstet Gynaecol Br Commonw 1972; 79: $782-7$.

[37] Lambers DS, Clark KE. The maternal and fetal physiologic effect of nicotine. Semin Perinatol 1996; 20: 115-26.

[38] Lichtensteiger W, Ribary U, Schlumpf M, Odermatt B, Widmer HR. Prenatal adverse effects of nicotine on the developing brain Prog Brain Res 1988; 73: 137-57.

[39] Kim SJ, Lee JH, Suh DC. Cerebellar MR changes in patients with olivary degeration: MR findings and pathologic findings. Radiology $1994 ; 192: 1715-9$.

(C) Lavezzi et al.; Licensee Bentham Open.

This is an open access article licensed under the terms of the Creative Commons Attribution Non-Commercial License (http://creativecommons.org/licenses/by-nc/3.0/) which permits unrestricted, non-commercial use, distribution and reproduction in any medium, provided the work is properly cited. 